\title{
SAÚDE, CONSUMO DE MEDICAMENTOS E COMUNICAÇÃO: UM ESTUDO SOBRE O USO DA INTERNET E DAS REDES SOCIAIS POR PESSOAS DE MAIS DE SESSENTA ANOS
}

Euripedes Oliveira Junior Fernandes*

RESUMO

Como a geração "Baby Boomer”, pessoas nascidas entre 1946 e 1964, utilizam a internet e redes sociais para se informar sobre saúde e compra de medicamentos no Brasil? Este artigo, de natureza exploratória, desperta a atenção para um conjunto de indagações relacionadas a saúde, e com a vida de uma parcela crescente da população do País. Os resultados apontam que a relação dos "Baby Boomers" com a tecnologia vem mudando, tornando-os ativos nas redes sociais e comunidades voltadas para a saúde, com o aumento do conhecimento e experiências com seus semelhantes graças a troca de informações.

Palavras-chave: Comunicação, Redes sociais digitais, Medicamentos.

\section{ABSTRACT}

How does the "Baby Boomers" generation, people that was born between 1946 and 1964, use the internet and social networks to inform about health and the purchase of medicines in Brazil? This exploratory article focus in questions related health, and to the lives of a growing portion of the population of the country. The results that the "Baby Boomers" relationship with technology, has been changing, making - active in social networks and communities focused on health, which favors an increase in and knowledge with their peers thanks to the exchange of information.

Keywords: Communication, Social networks. Medicines.

\section{INTRODUÇÃO}

Independentemente da idade, a adoção de tecnologias digitais na vida cotidiana pode representar um importante fator de inclusão social. Também para a chamada geração "Baby Boomer" - formada por pessoas nascidas

* https://orcid.org/0000-0002-4437-654X

Universidade Metodista 
após a Segunda Guerra Mundial, mais precisamente no período entre 1946 e 1964 e que, portanto, não são nativas desse universo -, essas tecnologias favorecem a compreensão de um mundo integrado e dinâmico, abrindo possibilidades de consumo e de relacionamentos. Nesse sentido, a popularização das redes sociais também transformou um conjunto de ferramentas em fonte de pesquisa sobre doenças e busca por medicamentos.

A expressão "baby boomer" possui uma história. Com o término da Segunda Guerra Mundial - é a história que se conta - os soldados dos países que participaram do conflito, ao retornarem a suas casas após longo período de ausência, teriam provocado um aumento nas taxas de natalidade, resultando em uma verdadeira explosão populacional após nove meses desse retorno. A esse fenômeno, então, se deu o nome de "Baby Boom" - "explosão de bebês", justificando desse modo o nome que iria ser dado a essa geração, "Baby Boomer", ou simplesmente "Geração Pós-Guerra".

Talvez o melhor, mesmo, para fugir ao folclore que pode estar cercando pelo menos em parte essa expressão - parece evidente o peso da narrativa de guerra dos Estados Unidos na construção da ideia - seja ficar com a noção, também reducionista, mas menos problemática, de "Geração Pós-Guerra", como apontam Souza e Gomes (2018). Não há clareza nem uma definição correta sobre os períodos que compreendem cada geração, como também não há segurança sobre para que de fato pode servir a divisão da história humana em períodos (GONÇALVES, 2018).

Neste estudo, para uma melhor compreensão de quais pessoas estamos falando, será considerado como pertencendo à geração "baby boomer" quem nasceu no período indicado e que tem hoje idade entre 54 a 77 anos, conforme Novelli, Leite e Sitta (2010). No Brasil, de acordo com o IBGE (2010), esta faixa etária representa cerca de $20,45 \%$ da população, com 39 milhões de pessoas, mais do que a população do Chile, Paraguai e do Uruguai juntos Countrymeters (2020).

No caso específico deste nosso estudo, cujo tema mais amplo é o da saúde dos Baby Boomers, a aplicação e o uso das redes sociais digitais são os mais diversos. Existem as buscas de informações sobre sintomas e tratamento das mais variadas doenças, com o risco de as respostas encontradas serem insuficientes, ambíguas ou não verdadeiras. É possível que se comprometa desse modo a saúde dos usuários, levando-os a consequências indesejáveis e ao agravamento de uma doença, uma vez que a utilização inadequada de 
medicamentos pode esconder determinados sintomas, causar reações alérgicas, dependência e até a morte.

Para este estudo, utilizamos o modelo exploratório de pesquisa, que, de acordo com Gil (2008 p. 27), tem como principal finalidade desenvolver, esclarecer e modificar conceitos e ideias, tendo em vista a formulação de problemas mais precisos ou hipóteses pesquisáveis para estudos posteriores. Conforme esse autor, esse tipo de pesquisa é realizado especialmente quando o tema escolhido é pouco explorado e torna-se difícil sobre ele formular hipóteses precisas e operacionalizáveis.

Sob essa perspectiva, explorar um tema significa reunir mais conhecimento sobre o mesmo e incorporar características inéditas, buscando dimensões até então não conhecidas.

Para tanto, realizamos um levantamento bibliográfico com base em estudos sobre a utilização das redes sociais como influenciadoras na compra de medicamentos pela geração "Baby Boomer". A pesquisa teórica permite ainda desvendar a perspectiva de qual é o impacto das redes sociais na compra de medicamentos pela geração "Baby Boomer", sob o ponto de vista da comunicação.

\section{UMA NOVA CULTURA E SEUS IMPACTOS NO COTIDIANO DAS PESSOAS}

Castells (2003), compara o fenômeno das tecnologias da informação ao que foi a eletricidade e o motor elétrico na Era Industrial, pela sua capacidade de distribuir a força da informação por todo o domínio da atividade humana. Há em todo esse processo complexo e multiangular - vista a coisa toda pelo viés de seus lados possivelmente positivos - uma evidente contribuição para mudanças na forma como as pessoas se relaciona entre si e com o mundo, favorecendo o aprendizado, novas habilidades, a capacidade cognitiva e a convivência social, que são essenciais para manter-se ativo, especialmente na idade mais avançada.

Pesquisas realizadas pelo Serviço de Proteção ao Crédito - SPC Brasil e pela Confederação Nacional de Dirigentes Lojistas - CNDL (2016) com pessoas acima de 60 anos, mapeou o estilo de vida dessa população e a sua relação com a tecnologia.

Os resultados revelaram que mais da metade desse público (53,9\%) acessa a Internet, 39\% dos quais diariamente, sendo o Smartphone o meio mais utilizado (61\%), seguido pelos computadores $(54 \%)$. 
No que diz respeito às Redes Sociais, ainda segundo a mesma pesquisa, 77\% deles utilizam o Facebook, 73\% WhatsApp e 39,8\% Youtube. Finalmente, somente $12 \%$ usam a Internet para fazer compras, e os produtos mais adquiridos são eletroeletrônicos (51\%), eletrodomésticos (43\%) e viagens $(42 \%)$.

Em outra pesquisa elaborada pela Confederação Nacional de Dirigentes Lojistas - CNDL (2018), foram entrevistados 612 consumidores com idade acima de 60 anos, de ambos os gêneros e de todas as classes sociais, nas 27 capitais brasileiras. Esta pesquisa revelou que 68\% desse público acessam a internet, dos quais $47 \%$ costumam ficar conectados todos os dias, com uma média de acesso de seis dias por semana; 63\% têm smartphone e 77\% dos acessos são feitos por esse dispositivo, seguido pelo computador (40\%), notebook $(30 \%)$ e tablet $(14 \%)$.

Segundo os entrevistados, os principais motivos para navegar na internet são: manter o contato com conhecidos (68\%), ficar informado sobre os principais assuntos que acontecem no Mundo (47\%), buscar informações sobre Produtos e Serviços (44\%), fazer Transações Bancárias (28\%), não ficar ultrapassado (21\%) e fazer compras (21\%).

Quanto aos itens mais adquiridos na internet, têm-se eletroeletrônicos $(60 \%)$, eletrodomésticos (56\%), viagens (43\%), livros (33\%), móveis $(30 \%)$, roupas $(30 \%)$ e remédios $(28 \%)$.

Em relação às redes sociais 84\% são usuários do WhatsApp, 68\% usam o Facebook e 41\% acessam o Youtube.

Finalmente, a pesquisa revelou que esse público busca aprender novas habilidades, estimular a capacidade cognitiva e cultivar a convivência social, ações essas consideradas essenciais para manter-se ativos nesta etapa do ciclo de vida dos respondentes; $\mathrm{E}$ a tecnologia vem contribuindo quanto às formas de se relacionar com as pessoas e com o mundo.

No contexto do público-alvo deste estudo, que busca permanecer ativo e vivo, Jenkins (2009 p. 47), menciona uma dimensão a ser apontada e que tem relação com comunidades de conhecimento que se formam em torno de interesses intelectuais mútuos; seus membros trabalham juntos para forjar novos conhecimentos, muitas vezes em domínios em que não há especialistas tradicionais; a busca e a avaliação de conhecimento são relações ao mesmo tempo solidárias e antagônicas.

As Redes Sociais Digitais, muito populares e utilizadas no Brasil -- sobre o que falaremos adiante -- bem no fundo, como se sabe, não constituem algo de fato original. 
Ao longo das nossas vidas, integramos grupos, estabelecemos relações com família, amigos, comunidades, colegas de trabalho e instituições. Essas redes abrangem relações de maior ou menor intensidade com o grupo de pares, família, escola, comunidade ou ainda com instituições ou associações relacionadas a esporte ou lazer, como música, dança entre outras.

O olhar de Martino (2015 p. 241) aponta para um processo pelo qual as relações humanas e as práticas sociais se articulam com as mídias, resultando em alterações no desenvolvimento dessas atividades, e de como as coisas acontecem quando essa articulação se dá.

Dentro desta linha de pensamento, Stig Hjarvardque chama de "mediatização" a centralidade que as mídias ocupam nas experiências cotidianas de uma parte considerável da população mundial. Segundo Hjarvardque (2012 p.64), "A mediatização é o processo pelo qual a sociedade em um grau cada vez maior está submetida a, ou torna-se dependente da mídia e da sua lógica."

É justo inferir, a partir do que aponta Stig Hjarvardque, que a Internet vem modificando de forma tangível os modos como os "Baby Boomers" organizam o seu consumo e como buscam se informar sobre os mais diferentes assuntos de seus interesses. Como corolário de todos esses processos de mudança, resulta que as Mídias Digitais se integraram às rotinas de outras instituições, como política, família, trabalho e religião. Assim, um número cada vez maior das atividades desses domínios institucionais é realizado por intermédio dos meios de comunicação interativos, o que reforça a ideia da transformação que vem acontecendo também com os "Baby Boomers".

Como o público mais jovem, os "baby boomer" também são inspirados pelos influenciadores digitais, e pesquisa realizada pelo Instituto Locomotiva, Webshoppers da e-Bit e números do Dynamic Big Data (2017 p. 6), intitulada "O x da questão", revelou que apenas 16\% desse público é influenciado diretamente pelos influenciadores digitais ( $3^{\text {a }}$ opção), sendo que $33 \%$ deles recorrem à decisão conjunta (primeira opção) e 27\% à decisão do lar (segunda opção), o que nos faz inferir que os influenciadores digitais ainda não são os grandes inspiradores do público-alvo desse estudo.

\section{AUMENTO DA ESPERANÇA MÉDIA DE VIDA NO BRASIL}

Em um passado recente, a população dos países europeus, da América do Norte e do Japão era tida como mais velha, sendo o Brasil associado a um país de população jovem. Mudou muito, e rapidamente, como se sabe. O Ipea (2018), no terceiro volume do seu relatório Desafios da nação, revela que a 
expectativa de vida aos 65 anos aumentou de 10,6 para 18,4 anos entre 1940 e 2015, ou seja, um incremento de 73,6\%. Na idade de 60 anos, no mesmo período, saltou de 13,2 para 22,1 anos (mais $67,4 \%$ ).

Esse mesmo relatório aponta que a projeção demográfica do IBGE, atualizada em 2013, estima que no Brasil a população de pessoas com 60 anos ou mais deve crescer, entre 2017 e 2060, em média, em 1,1 milhão de pessoas ao ano (a.a.), atingindo 73,6 milhões em 2060. Em termos de participação na população total, o patamar atual, na casa dos $10 \%$, deverá crescer para cerca de 33,7\% em 2060. De acordo com o Censo Demográfico de 2010, cerca de 1 em cada 9 brasileiros era idoso; em 2060, 1 em cada 3 brasileiros terá 60 anos ou mais de idade.

Pelos dados harmonizados da Pnad/IBGE, a participação das pessoas idosas no total da participação vem crescendo de forma acelerada, tendo passado de 7,9\%, em 1992, para 14,4\%, em 2015. Pelos dados da Pnad/IBGE, em 2015, já havia mais pessoas com 60 anos ou mais de idade no Brasil do que crianças de 0 a 9 anos.

Também ocorre um processo de envelhecimento da própria população idosa, com incremento da importância relativa das pessoas com 80 anos ou mais de idade. Enquanto a população total irá crescer ao ritmo de 0,1\% a.a. entre 2017 e 2060, as populações com 60 anos ou mais e 80 anos ou mais de idade irão crescer ao ritmo de $2,5 \%$ a.a. e 3,9\% a.a., respectivamente. Nessas projeções populacionais, junto ao aumento da importância relativa dos idosos, e até mesmo como efeito da queda de fecundidade, observa-se um estreitamento da base da pirâmide etária brasileira, com clara redução da participação dos grupos etários que constituem a maior parte da população economicamente ativa. O ápice da participação da população de 15 a 64 anos na população total, de acordo com a projeção demográfica do IBGE, está previsto para o ano de 2022, o que denota que o chamado bônus demográfico já se encontra em sua fase final no Brasil. ${ }^{1}$

O bônus demográfico é caracterizado pelo período em que, por causa da redução na fecundidade feminina, a estrutura populacional fica favorável ao crescimento econômico porque há, proporcionalmente, um maior número de pessoas em idade ativa aptas a trabalhar - ou seja, há menos crianças na população, e o percentual de idosos ainda não é elevado 
Por fim, a participação dos idosos na população total está na casa dos $10 \%,{ }^{2}$ conforme aponta o IBGE (2013), sendo que a população com mais de 55 anos de idade movimenta quase 20\% do Produto Interno Bruto (PIB), de acordo com a pesquisa IPSOS Flair (2018).

Essas projeções revelam uma presença cada vez maior dessa faixa etária na população, e com isso os estudos acerca desse público passam a ganhar maior relevância a partir da década de 1990. Para Moschis (2003), os estudos desse mercado e dos esforços de marketing podem ser divididos em três etapas, sendo uma anterior à década de 1980, outra durante a década de 1980 e a terceira, a partir dos anos 1990, período esse em que as empresas passam a compreender e a reconhecer esse mercado, desenvolvendo campanhas de marketing para atingí-lo e, inclusive, segmentá-lo. Convém notar que essa terceira etapa coincide com o período da implantação da internet comercial no Brasil.

A pesquisa IPSOS Flair, cunha, inclusive, a expressão "Me Generation” geração da "Autorrealização", qual seja a dos Baby Boomers, que vêm ocupar a nova terceira idade, pois gostam de ser reconhecidos pelo que já fizeram e por sua trajetória.

Portanto, para as marcas é fundamental focar em sua experiência, e não na idade que carregam.

\section{O PODER DAS REDES SOCIAIS}

As redes sociais, como adiantamos, são altamente utilizadas e populares no Brasil. Segundo pesquisa publicada no Canaltec (Tabela 1), estamos entre os principais usuários na América Latina. O Brasil tinha 78,1 milhões de usuários mensais ativos - e que acessaram uma rede social ao menos uma vez por mês em 2014, número que subiu para 86,5 milhões em $2015(+10,7 \%)$ e alcançou a metade de 2016 com 93,2 milhões de usuários (+ 7,8\%). Em segundo lugar está o México, que, para efeito comparativo, saltou de 43,7 milhões em 2014 para 49,5 milhões em 2015 (+13,2\%), tendo chegado em junho de 2016 com 56 milhões de usuários ativos (+13,1\%). Finalmente, a Argentina, que tinha 19,2 milhões em 2014, foi para 20,8 milhões em 2015 $(+8,5 \%)$, chegando em junho de 2016 com 21,7 milhões de usuários $(+4,2 \%)$.

\footnotetext{
2 Pela projeção demográfica do IBGE, em 2015 e em 2017, a participação das pessoas com 60 anos ou mais de idade na população total seria de, respectivamente, 11,7\% e 12,5\%. Pelos dados da Pesquisa Nacional por Amostra de Domicílios (Pnad) de 2015, realizada pelo IBGE, contudo, a participação dos idosos na população total já seria de 14,3\%, um patamar bem superior ao observado pela mesma pesquisa em 2004, que foi de $9,7 \%$, denotando o rápido envelhecimento populacional pelo qual o Brasil está passando.
} 


\begin{tabular}{|l|c|c|c|c|c|c|c|}
\hline & $\mathbf{2 0 1 4}$ & $\mathbf{2 0 1 5}$ & $\mathbf{2 0 1 6}$ & $\mathbf{2 0 1 7}$ & $\mathbf{2 0 1 8}$ & $\mathbf{2 0 1 9}$ & $\mathbf{2 0 2 0}$ \\
\hline Usuários de redes sociais (milhões) & & & & & & & \\
\hline Brasil & 78,1 & 86,5 & 93,2 & 97,8 & 100,6 & 103,1 & 105,2 \\
\hline México & 43,7 & 49,5 & 56 & 61,6 & 65,9 & 69,8 & 72,1 \\
\hline Argentina & 19,2 & 20,8 & 21,7 & 22,5 & 23,3 & 23,9 & 24,3 \\
\hline Outros & 69 & 81 & 89,3 & 95,7 & 102 & 107,7 & 111,9 \\
\hline América Latina & 210 & 237,8 & 260,1 & 277,8 & 291,8 & 304,4 & 313,6 \\
\hline Crescimento dos usuários de redes sociais (\% de mudança) & & & & & & & \\
\hline México & 16,7 & 13,2 & 13,1 & 10 & 7 & 6 & 3,3 \\
\hline Brasil & 12,5 & 10,7 & 7,8 & 5 & 2,8 & 2,4 & 2,1 \\
\hline Argentina & 9,9 & 8,5 & 4,2 & 3,8 & 3,4 & 2,6 & 2 \\
\hline Outros & 16,2 & 17,5 & 10,3 & 7,3 & 6,4 & 5,6 & 3,9 \\
\hline América Latina & 14,3 & 13,2 & 9,4 & 6,8 & 5 & 4,3 & 3 \\
\hline
\end{tabular}

Tabela 1 - Usuários de Redes Sociais na América Latina

Fonte: Canaltec, 2016

A RockContent Inteligência, em sua pesquisa Social Media Trends 2018, apresentou resultados relativos a hábitos de uso e comportamento das pessoas nas redes sociais, constatando que, em linhas gerais, 95\% dos usuários respondentes da pesquisa utilizam Facebook, 89,1\%, o Instagram, e 11,3\%, o Snapchat. Diariamente 38\% dos usuários acessam suas redes sociais, gastando quatro horas por dia navegando nessas redes. Embora utilizem bastante as redes, 31,7\% dos respondentes estão pensando em sair de alguma rede social. Sob o ponto de vista socioeconômico, 47,4\% são homens e 52,6\%, mulheres. Na faixa etária do público-alvo deste estudo, $15,3 \%$ dos respondentes têm 40 anos ou mais, sendo que 57\% dos respondentes estão na faixa etária entre 25 a 39 anos de idade.

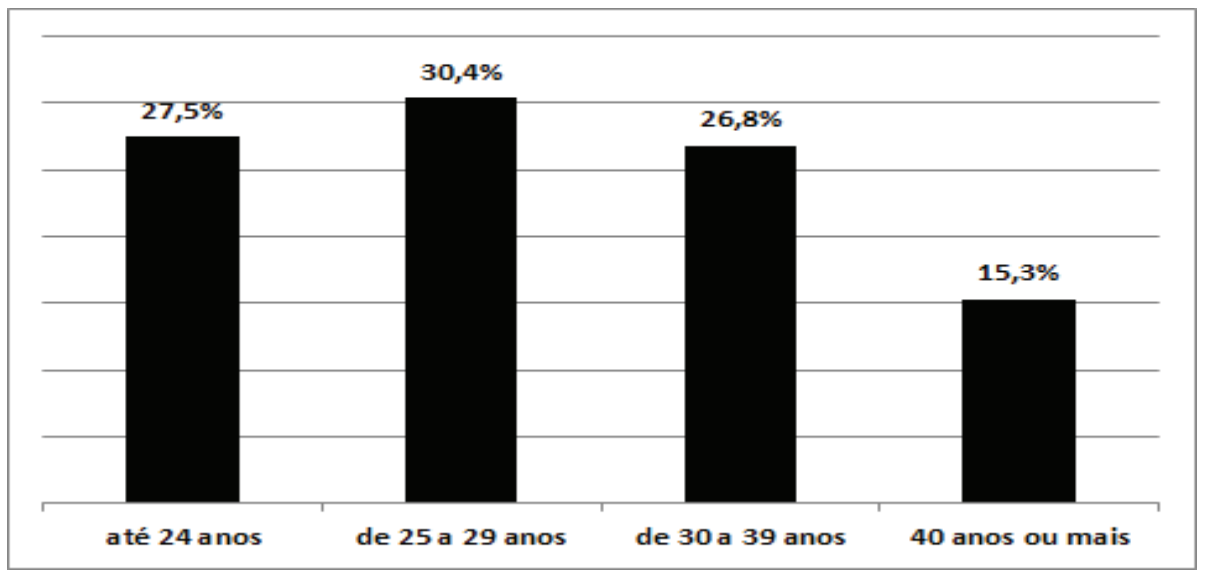

Gráfico 1 - Hábitos de Uso e Comportamento das Pessoas nas Redes Sociais Fonte: RockContent Inteligência - pesquisa Social Media Trends, 2018 
Quando se faz o cruzamento pela variável gênero, esta mesma pesquisa nos mostra (Tabela 2) que o Instagram é mais utilizado entre mulheres, sendo o Youtube e o Twitter entre homens; contudo, o Facebook é a rede social mais utilizada por todas as faixas etárias (exceto homens até 24 anos).

Já por faixa etária observa-se que, conforme a idade aumenta, diminui o percentual daqueles que usam o Instagram. É possível compreender também que o uso do Youtube e do Pinterest é menor entre aqueles com mais de 40 anos de idade (se comparado às demais faixas de idade). Por outro lado, a utilização do Twitter é maior nesse estrato. O LinkedIn, por sua vez, é mais utilizado entre respondentes da faixa de 30 a 39 anos. Por fim, o Snapchat alcança cerca de 19\% dos respondentes de até 24 anos de idade.

\begin{tabular}{c|c|c|c|c|c|c|}
\hline & HOMENS & MULHERES & ATÉ 24 ANOS & DE 25 A 29 ANOS & DE 30 A 39 ANOS & 40 ANOS OU MAIS \\
\hline FACEBOOK & $94 \%$ & $96 \%$ & $94 \%$ & $97 \%$ & $97 \%$ & $90 \%$ \\
\hline INSTAGRAM & $86 \%$ & $92 \%$ & $94 \%$ & $94 \%$ & $87 \%$ & $74 \%$ \\
\hline YOUTUBE & $73 \%$ & $66 \%$ & $77 \%$ & $66 \%$ & $71 \%$ & $59 \%$ \\
\hline LINKEDIN & $61 \%$ & $64 \%$ & $57 \%$ & $63 \%$ & $66 \%$ & $64 \%$ \\
\hline TWETtER & $45 \%$ & $36 \%$ & $40 \%$ & $35 \%$ & $41 \%$ & $49 \%$ \\
\hline PINTEREST & $29 \%$ & $50 \%$ & $42 \%$ & $42 \%$ & $40 \%$ & $31 \%$ \\
\hline GOOGLE+ & $21 \%$ & $18 \%$ & $13 \%$ & $9 \%$ & $24 \%$ & $45 \%$ \\
\hline SNAPCHAT & $12 \%$ & $11 \%$ & $19 \%$ & $12 \%$ & $6 \%$ & $4 \%$ \\
\hline
\end{tabular}

Tabela 2 - Cruzamento pela Variável Gênero

Fonte: RockContent Inteligência - pesquisa Social Media Trends, 2018

Quanto aos principais interesses nas redes sociais, este mesmo estudo nos mostra (Gráfico 2) os principais temas que os respondentes mais acompanham são: informações sobre sua profissão ou área de atuação (81\%), notícias em geral $(59,8 \%)$, entretenimento $(59,8 \%)$ e tecnologia $(56,7 \%)$. O interesse pela área de saúde, foco deste estudo, coloca-se como antepenúltima preferência de busca, com $23,5 \%$ das pesquisas. 


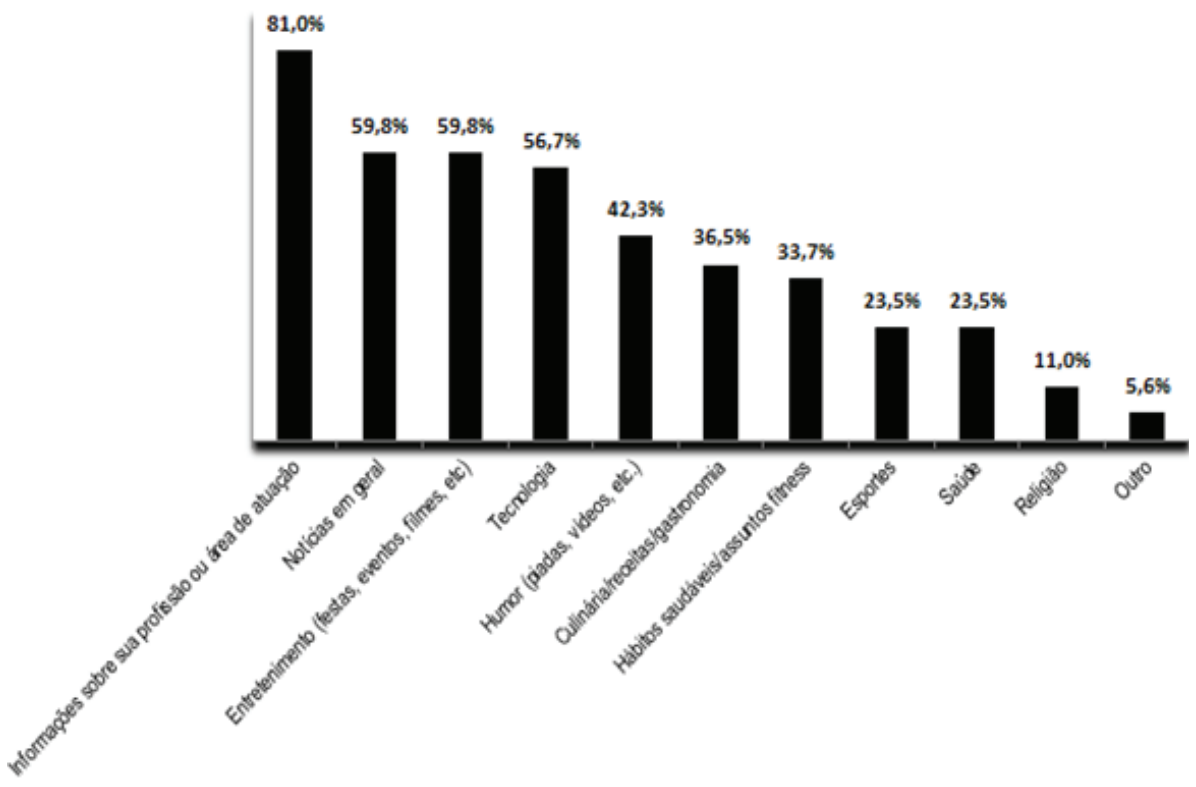

Fonte: KockContent Inteligènc1a - pesquisa Social Media 'Irends, 2018

4. BUSCA POR MEDICAMENTOS E SAÚDE NAS REDES SOCIAIS DIGITAIS

Conforme a iCrossing (2008), Agência de Marketing Digital, as redes sociais facilitam a troca de informações sobre saúde, compartilhamento de histórias pessoais que vão além de conteúdo médico ou conversa com amigos e familiares. O que esse estudo demonstrou é que as pessoas estão adotando as Redes Sociais Digitais nesse contexto, sendo que 1 em cada 3 americanos utiliza alguma rede social online, especificamente, para obter informações sobre saúde.

Nesse estudo, foi demonstrado (Gráfico 1) que, em termos de ferramentas online utilizadas para busca sobre informações de saúde, 58\% têm como base a internet (sites, motores de busca, anúncios, blogs, fóruns e redes sociais). 


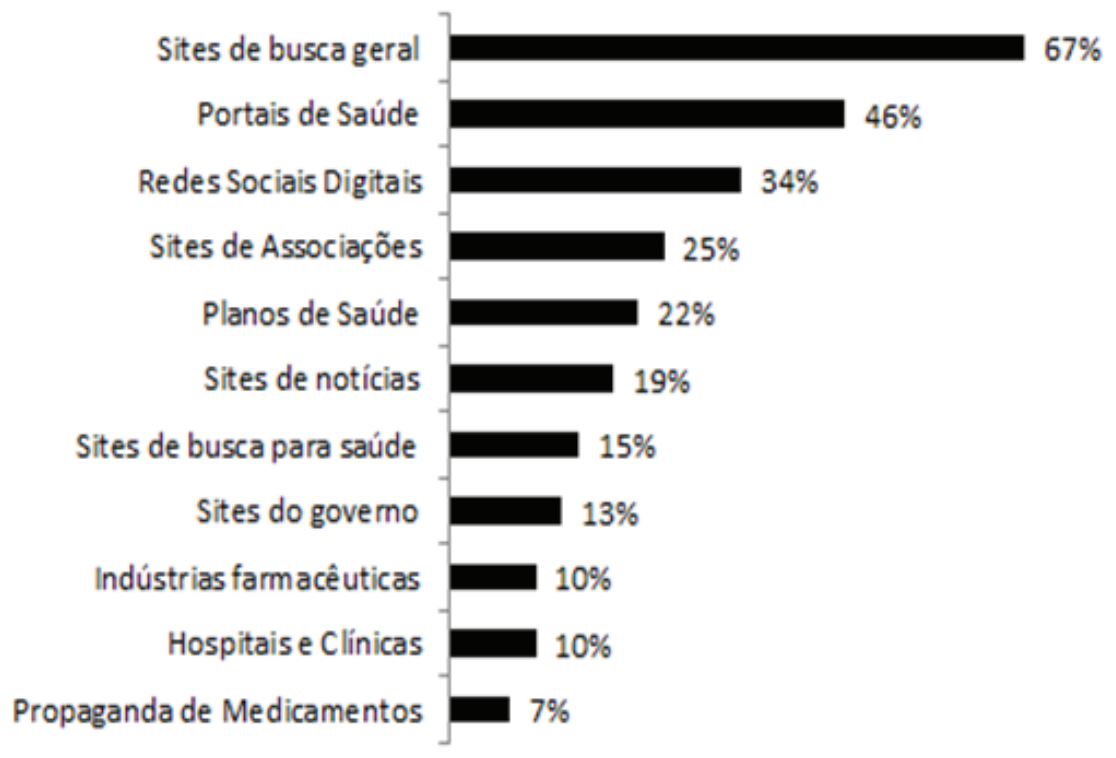

Gráfico 3 - Ferramentas Virtuais Utilizadas na Busca por Informações de Saúde Fonte: iCrossing (2008)

Há um número expressivo de sites e grupos, abertos e fechados, nas Redes Sociais Digitais voltados para a saúde, com troca de informações e experiências com os mais diversos temas e discussões.

O site Vida e Saúde (https://www.vidaesaude.orgL), por exemplo, traz informações sobre saúde, nutrição e bem-estar em diversas categorias, com temas atuais, oferecendo ainda cursos e conteúdo para consulta.

O Instituto Alzheimer Brasil (http://www.institutoalzheimerbrasil.org. br $\angle$ ), estimula o compartilhamento de experiências de familiares de portadores dessa doença degenerativa, com reuniões mensais em igrejas para discutir avanços na cura ou diminuição dos sintomas da doença, entre outras iniciativas de suporte aos familiares e pessoas portadoras da doença. Seus fundadores e corpo diretivo são profissionais com interface profissional e conhecimento sobre a doença, e é possível associar-se com base em código de conduta com regras e atribuições claras.

Já o portal Orphanet (https://www.orpha.net/consor/cgi-bin/index. php?lng=PT) reúne informações e promove o conhecimento sobre doenças raras de forma a melhorar o seu diagnóstico, cuidado e tratamento. A Or- 
phanet foi criada na França pelo Inserm (Instituto Nacional Francês para a Saúde e Investigação Médica), em 1997, e desde 2000 vem sendo apoiada por subvenções da Comissão Europeia, tornando-se um consórcio de 40 países na Europa e em todo o mundo.

Nesse contexto, insere-se o tema do autodiagnóstico. Com muita facilidade encontram-se informações sobre sintomas, profilaxia, medicamentos, tratamentos e as possíveis formas de contágio das mais diversas doenças. O site Toda Biologia (https://www.todabiologia.com/doencas/) traz várias doenças, seus sintomas, causas e fatores desencadeantes.

Há ainda associações que mantêm áreas restritas a profissionais de saúde e também ao público-leigo, como a Sociedade Brasileira de Diabetes (http:// www.diabetes.orb.br) e o Instituto Nacional do Câncer (http://www.inca.gov. br), que disponibilizam informações sobre doenças, suas respectivas causas e medidas preventivas, entre outras.

Por sua proporção e diversidade, as informações na área de saúde obtidas na internet podem ser de baixa qualidade, ter origem duvidosa, ser insuficientes, estar desatualizadas ou contar com pouca ou nula base científica.

Uma busca no Google, realizada em agosto de 2019, utilizando o termo "Diabetes", trouxe aproximadamente 526.000.000 de resultados, encontrados em ambientes que vão desde sites (jornais, educacionais, acadêmicos, hospitais, clínicas e consultórios), passando por grupos de autoajuda organizados por pessoas diagnosticadas com determinada condição ou doença e, ainda, comunidades virtuais, redes sociais e fóruns de discussão.

Nenhum desses ambientes virtuais é ou foi avaliado antes de ser disponibilizado na internet, conforme Mendonça e Neto (2015), no artigo "Critérios de avaliação da qualidade da informação em sites de saúde: uma proposta”.

De tal forma que essas informações podem estar incompletas, incorretas ou serem incompreensíveis para um leigo. Considerando os riscos que informações encontradas na internet oferecem, especialistas em informação vêm desenvolvendo critérios e indicadores, na tentativa de estabelecer padrões mínimos de qualidade, como aponta Lopes (2008 p. 2).

Há indicadores que ampliam, enquanto outros diminuem a qualidade da informação na Web. Para Fogg e Cols (2002), um desses indicadores está relacionado ao domínio do site como os domínios: governamental (.gov), educacional (.edu) ou institucional (.org) trazem no seu bojo maior reconhecimento, ao contrário do site pessoal. Poderá haver maior credibilidade 
se houver endereço que permita ao usuário acesso ao gestor da página para, por exemplo, ter suas demandas atendidas, conforme esses mesmos autores.

Dentro dessa mesma linha de pensamento, para Mostafa e Terra (1998), grande parte dos critérios obedece a um consenso sobre os questionamentos básicos relacionados com o processo de comunicação científica, ou seja, valorizam a relevância, autoridade, objetividade, atualização e a cobertura do site.

Eysenbach e colaboradores (2002) examinaram 79 estudos que avaliaram a qualidade da informação em sites de saúde. Esse estudo revelou cinco critérios de qualidade mais frequentemente utilizados, quais sejam:

1) Técnico: refere-se à questão de como a informação foi obtida e disponibilizada, constituindo variações do que poderia ser chamado de critério de credibilidade, transparência e ética profissional;

2) Acurácia: mensura o grau de concordância da informação fornecida com a melhor evidência ou com a prática médica;

3) Abrangência: observa o grau de cobertura da informação;

4) Inteligibilidade: verifica o nível de complexidade e dificuldade de leitura do texto;

5) Design: avalia aspectos visuais do site.

\section{SISTEMAS DE CERTIFICÃO E OU ACREDITAÇÃO DE QUALIDADE}

Mendonça e Neto (2015) observam que alguns países desenvolveram sistemas de certificação ou acreditação de qualidade da informação com rigoroso processo de análise, entre os quais se destaca a participação de especialistas que garantem a qualidade de informação de um site. Os Estados Unidos contam com o maior número de iniciativas na área:

Uma das mais tradicionais é a Agency for Healthcare Research and Quality (AHRQ), que atua desde 1997. Sua missão é produzir evidências para tornar o cuidado em saúde equitativo, mais seguro e acessível e com melhor qualidade, por meio do Departamento de Saúde e Serviços Humanos dos Estados Unidos;

A American Medical Association (AMA) desenvolveu normas de qualidade com o objetivo de manter o controle de qualidade dos sites de seus associados, tendo publicado o Guidelines for medical and bealth information sites on the Internet, ou AMA Guidelines;

A Internet Healthcare Coalition (IHC), que por sua vez é uma organização sem fins lucrativos que reúne seguradoras de saúde nos Estados Unidos 
(EUA) desde 1997. Sua função principal é fornecer alternativas acessíveis de cuidados de saúde pela internet. Em 2000, a IHC elaborou um código de ética no qual apresenta oito princípios para sites de saúde. O eHealth code of ethics visa garantir que pessoas possam confiar na informação disponível e tenham plena compreensão dos riscos inerentes à adoção desta ou daquela prática;

A Accreditation Healthcare Commission, organização sem fins lucrativos fundada em 1990, criou, em 2001, o sistema de credenciamento de sites, denominado Utilization Review Accreditation Commission (URAC), que verifica cerca de 50 itens de qualidade para credenciar sites de saúde. O processo de acreditação é iniciado, após a solicitação do requerente, por um comitê de acreditação e revisão, que inclui especialistas de diversas áreas. Um requisito importante desse programa de acreditação é que o requerente deve demonstrar que estabeleceu um comitê de qualidade para supervisionar anualmente a conformidade com os padrões.

Na Europa destaca-se a Comissão da Comunidade Europeia. Essa organização continental publicou o documento intitulado Health Literacy and Internet: Recommendations to Promote Health Literacy by the Means of the Internet, em que estabelece um conjunto de critérios de qualidade para uso dos Estados-membros e para o público em geral. O objetivo principal foi orientar autoridades nacionais e regionais, associações de profissionais de saúde e instituições que disponibilizam suas páginas na web a seguirem os critérios de qualidade propostos.

Em relação à orientação aos usuários, duas iniciativas europeias chamam mais a atenção: uma inglesa e outra francesa. Em 1999, o Instituto de Ciências da Saúde na Inglaterra da British Library \& University Oxford criou o Discern, composto de um formulário online com 15 questões. Organizadas em três seções, as questões foram desenvolvidas especialmente para que os indivíduos que procuram informação sobre tratamentos avaliem se a informação disponível no site consultado é ou não confiável e abrangente. Além disso, existem outras iniciativas na Inglaterra, França e Espanha.

A Associação Francesa Centrale Santé, inspirada no Código de ética da Internet Healthcare Coalition" (IHC), elaborou o NET Scoring, incorporando alguns aspectos relacionados com a comunidade francesa no contexto europeu. Para garantir a máxima objetividade na seleção dos critérios, a Associação formou um grupo multidisciplinar que incluiu profissionais de saúde, engenheiros, bibliotecários, advogados e representantes de algumas organizações profissionais, os quais definiram questionário com 49 critérios, 
divididos em oito categorias. Cada critério recebe um peso específico, a saber: critério essencial (peso de zero a nove); critério importante (peso de zero a seis) e critério menor (peso de zero a três). O total desses critérios atribuído a uma página web fornece a respectiva medida de desempenho dessa página.

A Fundação Health On the Net (HON), sediada na Suíça, é uma da mais antigas e conhecidas iniciativas de avaliação da qualidade da informação sobre saúde na internet. A HON atribui um selo de qualidade para os sites de saúde que estejam em conformidade com os seus princípios. Os sites certificados são revistos, anualmente, para ratificar ou não o credenciamento em atendimento aos princípios estabelecidos. A instituição fornece ainda um código de conduta para orientar os usuários a avaliarem se um determinado site está em conformidade com seus princípios. Para tanto, dispõe de um vídeo instrucional para ajudar aos usuários identificar sites confiáveis.

Na Espanha, a Web Médica Acreditada (WMA), programa de certificação vinculado ao Colégio Oficial de Médicos de Barcelona (COMB), oferece um selo de qualidade que identifica os sites que atendem a determinados requisitos em termos de qualidade de conteúdo e confiabilidade dentro da indústria médica e de saúde do país. Outra iniciativa espanhola vem da Andaluzia. Trata-se da Agencia de Calidad Sanitaria de Andalucía, que desenvolveu o Programa de Acreditación de Páginas Web Sanitárias. Baseado nas recomendações da iniciativa da Comissão Europeia, o programa pretende verificar o nível de confiança que as páginas web têm em relação ao conteúdo, conectividade e aos serviços que oferecem aos seus usuários. A agência também propõe um selo de confiança, concedido por meio do mencionado programa, que define diferentes níveis de acreditação, a saber: avançado, bom ou excelente. Esses níveis indicam o grau de conformidade com os grupos de critérios atendidos. A validade do selo atribuído ao site avaliado é de dois anos.

No Brasil o Conselho Federal de Medicina - CFM (2018) estabeleceu duas resoluções relacionadas à ética médica na publicidade e nas redes sociais, quais sejam: $\mathrm{n}^{\circ} \mathrm{s} 1.974 / 11$ e $\mathrm{n}^{\circ} 2.126 / 15$, que delineiam claramente o que pode e o que não pode ser feito nessa área, estabelecendo a zona proibida do sensacionalismo, autopromoção e da concorrência desleal. Há iniciativas com manuais de conduta para mídias sociais publicadas pelas Sociedades, como, por exemplo, a Sociedade Brasileira de Anestesiologia - SBA (2017, que orienta e busca estabelecer parâmetros para aquilo que é permitido ou proibido, sob o ponto de vista destas instituições, para os profissionais das distintas especialidades médicas.

A Anvisa - Agência Nacional de Vigilância Sanitária, agência reguladora, sob a forma de autarquia vinculada ao Ministério da Saúde exerce o controle 
sanitário de todos os produtos e serviços (nacionais ou importados) submetidos à vigilância sanitária, tais como medicamentos, alimentos, cosméticos, saneantes, derivados do tabaco, produtos médicos, sangue, hemoderivados e serviços de saúde, não estabelecendo normas ou resoluções específicas para questões relacionadas à publicação de conteúdo na internet ou redes sociais no Brasil.

Mesmo no Brasil, ainda com algumas iniciativas, e acompanhando a tendência de países Europeus e Estados Unidos no que se refere a chancela de informações a serem publicadas na Internet e Redes Sociais Digitais.

Um fenômeno recente, relacionado aos influenciadores digitais, que nasceram e têm crescido a partir das Redes Sociais Digitais, também se faz presente no segmento de saúde. O Centro de Comunicação Digital - Cecom e a Revista Negócios da Comunicação, em conjunto com a Agência Air Influencers, que por meio de métricas de audiência, influência e relevância, selecionou seis dos melhores influenciadores digitais de 22 diferentes categorias - entre eles, aqueles da área de saúde, para o Prêmio Influenciadores Digitais 2019 http://premioinfluenciadores.com.br/premio-votacao/. Todo este processo foi auditado pela agência DBO Brasil.

\subsection{Riscos e o Perigo da Automedicação}

Há uma indagação inquietante a ser respondida e que diz respeito às razões pelas quais as pessoas recorrem às redes sociais digitais na área de saúde. A Tabela 3 apresenta algumas delas por ordem de importância.

\begin{tabular}{l|c|}
\hline Para ver o que outros pacientes dizem a espeito do remédio ou do tratamento & $36 \%$ \\
\hline Para pesquisar o conhecimento e experiencia de outros pacientes & $31 \%$ \\
\hline Para aprender habilidades ou obter treinamento que me ajude a lidar com a minha condição da doença & $27 \%$ \\
\hline Para obter apoio emocional & $17 \%$ \\
\hline Para construir uma conciência em torno da doença ou de sua causa & $15 \%$ \\
\hline Para compartilhar meu conhecimento e experiência sobre o remédio ou tratamento da minha doença & $14 \%$ \\
\hline Para compartilhar o meu conhecimento e experiêcnia sobre uma questão de saúde & $14 \%$ \\
\hline Para encontrar recomendações de pacientes e opiniões sobre hospitais e outras opções de tratamento & $13 \%$ \\
\hline Para encontrar recomendações de pacientes e opiniões sobre hospitais e outras centros de tratamento & $13 \%$ \\
\hline Para encontrar recomendaçoes de pacientes e opiniões sobre médicos & $10 \%$ \\
\hline Para sentir que pertenço a um grupo ou comunidade & $8 \%$ \\
\hline Nenhuma acima & $22 \%$ \\
\hline
\end{tabular}

Tabela 3 - Razões de utilização das redes sociais de saúde Fonte: The Sociology of Caregiving. p.202 
Outra faceta dessa discussão diz respeito a possíveis barreiras pelas quais as pessoas compartilham informações nas redes sociais digitais e que estão relacionadas à própria privacidade, sendo que os dados relativos à saúde são pessoais, e há preocupação em compartilhar esse tipo de informação no universo online.

Há um princípio na administração de medicamentos que todos os enfermeiros conhecem, e que é muito enfatizado durante a formação desses profissionais, denominado o princípio dos cinco certos, ou seja: que o medicamento certo seja dado ao paciente certo, na dose certa, na via certa e no horário certo (CASSIANI, 2005).

Para Klaassen (1985), "a posologia correta diferencia o veneno do remédio”. Figueiredo, Bonaina e Ortiz (2011) fazem referência à Conferência Mundial sobre Uso Racional de Medicamentos, em Nairóbi, capital do Quênia, onde foi reconhecida a existência da automedicação de forma racional, e que de acordo com a definição proposta, o uso adequado de medicamentos ocorre quando o indivíduo os obtém de acordo com suas necessidades clínicas, em doses corretas, por período de tempo apropriado e com baixo custo.

No Brasil, a Constituição Federal de 1988 atribui ao Estado a responsabilidade de garantir saúde à população, através do acesso a bens e serviços, além da adoção de medidas de redução de riscos a doenças e agravos. $\mathrm{Na}$ Política Nacional de Medicamentos (PNM), os gestores são responsáveis pela Promoção do Uso Racional de Medicamentos (BRASIL, 1988, 1998). O Uso Racional de Medicamentos (URM) foi definido como o processo que considera a prescrição apropriada, disponibilidade oportuna, dispensação adequada e consumo em doses, intervalos e períodos indicados de medicamentos eficazes, seguros e de qualidade (BRASIL, 1988).

O URM é uma resultante de interações entre diferentes atores sociais, com responsabilidades e atribuições de governos, de profissionais de saúde e da sociedade para a sua efetivação (COELHO; PINHEIRO; MAGARINOS-TORRES, 2014). Em nível municipal, compete ao gestor disponibilizar os recursos e subsídios necessários à implementação e efetivação do uso mais adequado dos medicamentos, na sua área de atuação.

Essas condições, contudo, dependem especialmente do usuário, que deve respeitar doses, horários, e as restrições recomendadas. Na prática, a automedicação acontece de maneira descontrolada, e grande parte dela ocorre sem orientação médica ou acompanhamento de um profissional da área de 
saúde, sendo que muitas vezes o usuário não tem conhecimento suficiente para seguir os princípios da automedicação segura.

Estudos realizados por Kessler (1991), Farley (1995), Marwick (1997), citados por Fanhani et al. (2006) "mostram que 30\% a 50\% dos pacientes não usam os medicamentos conforme a prescrição, devido à ausência de informações adequadas".

Por outro lado, o Serviço Nacional de Informações Tóxico-Farmacológicas - Sinitox estima que a automedicação seja a causa mortis de aproximadamente 20 mil pessoas ao ano no país. Alguns estudos apontam que 10\% a $20 \%$ do total de casos de internações hospitalares nos Estados Unidos tenham os efeitos adversos de medicamentos como agente causal (MIRANDA, 2012).

\section{CONSIDERACÕES FINAIS}

Este artigo se propôs a analisar o tema da saúde e comunicação, sob o ponto de vista do impacto das redes sociais na compra de medicamentos pela geração "baby boomer". As discussões apresentadas demonstraram que essa faixa etária da população brasileira é crescente, robusta, estando presente e ativa nas redes sociais e comunidades voltadas para a saúde, trocando informações, o que favorece um aumento de conhecimento e experiências com seus semelhantes, trazendo reciprocidade e "valor" com benefício mensurável, qual seja: ao longo do tempo as experiências se consolidam não só pelo volume, mas também pela qualidade.

A relação dos "Baby Boomers" com a tecnologia vem passando por mudanças importantes, sendo adotadas cotidianamente por uma fatia significativa desse público, seja pelo acesso à internet, seja pela utilização das redes sociais digitais, seja pelo consumo. Caem por terra, portanto, argumentos utilizados quanto à dificuldade em acessar, comunicar, compreender, segmentar e engajar esse público, em consequência da mudança de estereótipo do "Baby Boomer" que cresceu, se tornou antenado e que utiliza as redes sociais digitais para cultivar a convivência social, conhecer novas habilidades, manter-se ativo e como uma "janela para o mundo" que oferece autonomia e vida própria.

Não é o escopo deste estudo compreender os influenciadores digitais, mas por conta da linguagem e da autoconfiança, e de serem "pessoas como eu", que vivem juntamente com seus familiares e amigos as agruras, ansiedades, expectativas, frustrações e que são distantes de uma autoridade governamental, dos negócios, da mídia e até um médico, quer nos pareceres 
por algumas destas razões que as pessoas valorizam e tendem a confiar nas informações obtidas através desse canal de comunicação.

Mesmo não sendo o escopo deste trabalho, mas dentro do tópico influenciadores digitais, foi possível identificar que a área de saúde também se faz presente e relevante neste universo. Neste contexto, este estudo revelou também que há uma crescente preocupação com a qualidade da informação na área de saúde em relação ao que é publicado na internet e redes sociais digitais no Brasil, com iniciativas ainda localizadas e bem estruturadas que buscam estabelecer padrões e direcionamentos claros do que pode ou não ser publicado, conforme iniciativas nos EUA e Europa, de forma mais consistente.

Podemos depreender que, mesmo sendo formadas por leigos, algumas dessas comunidades que fazem emergir os influenciadores digitais na área de saúde, sendo, portanto, vetores de informação, possivelmente devam cada vez mais oferecer informações de credibilidade, idôneas e comprovadas, sob pena de se tornarem irrelevantes por conta da velocidade e do alcance de tudo o que circula dentro das redes sociais digitais e internet.

As pesquisas realizadas neste estudo não respondem a todas as perguntas acerca do tema saúde e comunicação, sob a ótica do impacto das redes sociais na compra de medicamentos pela geração "baby boomer", mas contribuem de algum modo, apresentando evidências da rápida transformação pela qual passa esse público, se transformando desse modo em um embrião para futuros estudos.

\section{REFERENNCIAS}

BRUHN, John G.; REBACH, Howard M. The Sociology of Caregiving. Dordrecht: New York: Springer, 2014, p. 202.

CASTELS, M. A Galáxia da Internet. Editora Zahar. 2003.

CASSIANI, S.H.B. A segurança do paciente e o paradoxo no uso de medicamentos. Rev. bras. enferm. 2005, vol.58, n.1, pp.95-99. ISSN 0034-7167. Disponível em: <http://www.scielo.br/pdf/reben/v58n1/ a19.pdf>. Acesso em: 16/08/2020.

COELHO, H. L. L.; PINHEIRO, R. M.; MAGARINOS-TORRES, R. Promoção do Uso Racional de Medicamentos. In: OSÓRIO-DE-CASTRO, C. G. S. et al. Assistência farmacêutica: gestão e prática para profissionais da saúde. Rio de Janeiro: Fiocruz, 2014. p. 283-294.

Coleção IPSOS Flair. Brasil 2018: Caiu a Máscara! A hora da Verdade. Disponível em: <https://www. ipsos.com/sites/default/files/ct/publication/documents/2018-04/flair_brasil_2018-pt.pdf.> Acesso em 11/09/2020. p - 129 .

Confederação Nacional de Dirigentes Lojistas - CNDL. Pesquisa Terceira Idade 2018. Disponível em: <https://www.spcbrasil.org.br/pesquisas/pesquisa/5290.> Acesso em: 14/09/2020. 
Conselho Federal de Medicina. Ética Médica na Publicidade e nas Redes Sociais. Disponível em: $<$ http:/ / portal.cfm.org.br/index.php?option=com_content\&view $=$ article\&id=27359:2018-01-05-13-39-56\&catid=46. $>$ Acesso em 26/08/2020.

CONSTANZI, N.R.; AMARAL, A.D.; DIAS, C.R.; ANSILIERO, G.; AFONSO, L.E; SIDONE, O. J. G. Reforma da Previdência Social, p. 4-5, 2018. Disponível em: <http://repositorio.ipea.gov.br/bitstream/11058/8465/1/Reforma\%20da\%20previd\%C3\%AAncia\%20social.pdf.> Acesso em: 14/09/2020.

\section{Cruzamento pela Variável Gênero}

Disponível em: <https://cdn2.hubspot.net/hubfs/355484/Ebooks\%20MKTC/Social\%20Media\%20 Trends\%202018.pdf?t=1542483912554\&utm_source=hs_automation\&utm_medium=email\&utm_content $=39460531 \&$ \&hsenc=p2ANqtz-_Dxh1Jyn_ij4kjeVhXTQ_nUlhQXEJowLW9xGjc7A5F_ct18q95j0xl3YWk-uwDVFl01YqLcGPoDLKcycymEKAEeJ8xxw\&_hsmi=39460531>. Acesso em: 14/09/2020.

EYSENBACH, G. et al. Empirical Studies Assessing the Quality of Health Information for Consumers on the World Wide Web: A Systematic Review. Journal Am Med Assoc. 2002, 287(20): 2691-2700. Disponível em: <http://citeseerx.ist.psu.edu/viewdoc/download?doi=10.1.1.15.2721\&rep=rep1\&type=pdf. $>$ Acesso em 24/08/2020.

FANHANI, H. R. et al. Avaliação Domiciliar da Utilização de Medicamentos por Moradores do Jardim Tarumã, Município de Umuarama - Pr. Paraná: Arq. Ciênc. Saúde Unipar, Umuarama, v. 10, n. 3, p. 127-131, 2006. Disponível em: <http://webcache.googleusercontent.com/search?q=cache:F_ P2lfHqULEJ:revistas.unipar.br/index.php/saude/article/download/ $615 / 532+\& \mathrm{~cd}=1 \& \mathrm{hl}=\mathrm{pt}-$ -BR\&ct=clnk\&gl=b.> Acesso em: 20/04/2020.

Ferramentas Virtuais Utilizadas na Busca por Informações de Saúde

Disponível em: <http://api.ning.com/files/aQ*9UpEJWIfxu4YcpKwCrqAlruSAvElSuXvRBd2v4c5rY4 MnaNkuPE96hQp*JxAkduKxQzK4pioEMN7DfQKEGVOGrMiaCd3W/SearchingforHealth2008.pdf. > Acesso em: 09/09/2020.

FIGUEIREDO, M. C.; BONACINA, C. M.; ORTIZ, F. T. Armazenagem de Medicamentos em Domicílios pelos Moradores do Bairro Figueirinha, em Xangri-lá, RS. Rio Grande do Sul: Universidade Federal do Rio Grande do Sul - UFRGS, 2011. 22 p. 2011. Disponível em: < http://www.lume.ufrgs.br/ handle/10183/35597.> Acesso em: 14/09/2020.

Fundação Oswaldo Cruz. Clipping Internet. Abr. 2015. Disponível em: <https://sinitox.icict.fiocruz. br/sites/sinitox.icict.fiocruz.br/files//Clipping\%20Internet\%20Mar2015.docx.> Acesso em: 12/09/2020.

FOGG, BJ et al. Stanford-Makovsky Web Credibility Study 2002: Investigating What Makes Web Sites Credible Today - A Research Report by the Stanford Persuasive Technology Lab \& Makovsky \& Company.

[S.1.]: Stanford University [Internet]. 2002. [cited 2012 Mar 15] Disponível em: < http://credibility.stanford. edu/pdf/Stanford-MakovskyWebCredStudy2002-prelim.pdf.> Acesso em 24/08/2019

GIL, A.C. Métodos e Técnicas de Pesquisa Social. São Paulo. Editora Atlas, 2008.Disponível em: <https://ayanrafael.files.wordpress.com/2011/08/gil-a-c-mc3a9todos-e-tc3a9cnicas-de-pesquisa-social. pdf.> Acesso: 08/09/2020

GONÇALVES, Márcio Souza. Comunicação, Cultura e Subjetividade: Perspectivas de Análise. In: Encontro Anual da Compós, 27, 2018, São Paulo, SP. Anais (on-line). Disponível em: http://www.compos.org.br/ data/arquivos_2018/trabalhos_arquivo_W7B3MDOPUKPAMSJZHRYP_27_6635_24_02_2018_15_59_04. pdf.> Acesso em: 08/09/2020. 


\section{Hábitos de Uso e Comportamento das Pessoas nas Redes Sociais}

Disponivel em: <https://cdn2.hubspot.net/hubfs/355484/Ebooks\%20MKTC/Social\%20Media\%20 Trends\%202018.pdf?t=1542483912554\&utm_source=hs_automation\&utm_medium=email\&utm_content=39460531\&_hsenc=p2ANqtz-_Dxh1Jyn_ij4kjeVhXTQ_nUlhQXEJowLW9xGjc7A5F_ct18q95j0xl3YWk-uwDVFl01YqLcGPoDLKcycymEKAEeJ8xxw\&_hsmi=39460531.> Acesso em: 14/09/2020.

Hábitos dos 50+ - Um estudo de como os Maduros Consomem. MindMinders. Disponivel em: $<$ https://rdstation-static.s3.amazonaws.com/cms/files/18283/1521748472or_maduros_mindminers_v2.pdf.> Acesso em: 12/08/2020.

How the America Searches: Health and Wellness. Disponível em: <http://api.ning.com/files/aQ*9UpEJWIfxu4YcpKwCrqAlruSAvElSuXvRBd2v4c5rY4MnaNkuPE96hQp*JxAkduKxQzK4pioEMN7DfQKEGVOGrMiaCd3W/SearchingforHealth2008.pdf.> Acesso em 08/09/2020

Instituto Brasileiro de Geografia e Estatística - IBGE. Pesquisa Nacional por Amostra de Domicílios Contínua - PNAD Contínua. Disponível em: <https://sidra.ibge.gov.br/Tabela/1209\#resultado Acesso em: 12/09/2020.

JENKINS, H. A Cultura da convergência. São Paulo: Editora Aleph, 2009. Disponivel em: <https:// www.nucleodepesquisadosex-votos.org/uploads/4/4/8/9/4489229/cultura_da_convergencia_-_henry_jenkins.pdf.> Acesso: 09/11/2019.

KLAASSEN, C.D. Princípios de toxicologia. In: GILMAN, A.G. et al. Goodman e Gilman: as bases farmacológicas da terapêutica. 7. ed. Rio de Janeiro: Guanabara Koogan, 1985. p.1045-1052.

LOPES, IL. Novos paradigmas para avaliação da qualidade da informação em saúde recuperada na Web. Ciência da Informação. 2004;33(1): 81-90, 2004.

MARTINO, L.M.S. A vida mediada: Sonia Livingstone e “A Mediação de Tudo". In: Teoria das Mídias Digitais: linguagens, ambientes e redes. 2. ed. Petrópolis: Vozes, 2015, p. 243-246.

MARTINO, L.M.S. Cultura da convergência. In: Teoria das Mídias Digitais: linguagens, ambientes e redes. 2. ed. Petrópolis: Vozes, 2015, p. 34 a 39.

MAYFIELD, A. What is Social Media? New York: iCrossing, 2008. Disponível em: <https://www.icrossing.com/uk/sites/default/files_uk/insight_pdf_files/What $\% 20$ is $\% 20$ Social $\% 20$ Media_iCrossing_ebook. pdf $>$. Acesso em: 14/09/2020.

MELO, D. O.; RIBEIRO E.; STORPIRTIS S. A importância e a história dos estudos de utilização de medicamentos. Revista Brasileira de Ciências Farmacêuticas, São Paulo, n.4, out./dez., 2006 , p. 476-485. Disponível em: <http://www.lume.ufrgs.br/handle/10183/35597.> Acesso em: 13/09/2020.

MENDONÇA, A.P.B.; Neto, A.P. Critérios de Avaliação da Qualidade da Informação em Sites de Saúde: Uma Proposta. RECIIS - Rev Eletron de Comun Inf Inov Saúde. 2015 jan-mar; 9(1) | [www.reciis.icict.fiocruz.br] e-ISSN 1981-6278. Disponível em: <https://www.arca.fiocruz.br/bitstream/ icict/17022/2/6.pdf > Acesso em 27/08/2020.

MIRANDA, Conceição Pacheco; VIEIRA, Francisco de oliveira. O Risco da Automedicação: Informação em Prol da Mudança de Hábito. Disponível em: < https://docplayer.com.br/20237594-Risco-da-automedicacao-informacao-em-prol-da-mudanca-de-habito.html >.Acesso em: 20/04/2020.

MOSCHIS, G. Gerontographics. Journal of Consumer Marketing, v. 10, n. 3, p, 43 - 53. 1993.

MONTEIRO, E. R.; LACERDA, J. T. Promoção do Uso Racional de Medicamentos: Uma Proposta de Modelo Avaliativo da Gestão Municipal. Rio de Janeiro, v. 40, n. 111, p. 101-116, OUT-DEZ 2016. Disponível em: <http://www.scielo.br/pdf/sdeb/v40n111/0103-1104-sdeb-40-111-0101.pdf.> Acesso em: 28/08/2020. 
MOSTAFA, S. P.; Terra, M. As fontes eletrônicas de informação: novas formas de comunicação e de produção do conhecimento. SP em Persp. 1998 out./dez., 12(4): 1-12.

NEGRI, J.A; Araújo, B.C; BACELETTE, R. Desafios da Nação: Artigos de Apoio, Volume 2. - Brasília: Ipea, 2018. v. 2, capítulo 20. Disponível em: <http://www.ipea.gov.br/portal/images/stories/PDFs/ livros/livros/180327_desafios_da_nacao_apoio_vol2.pdf.> Acesso em: 20/08/2020.

NOVELLI, Valeria Aparecida Moreira; LEITE, Marilda Corrêa; SITTA, Maria Isabel Uthman. Mediação da Informação: Usuários Gerações Veteranos, Baby Boomers, X, Y, e Z. II Seminário Internacional de Bibliotecas Digitais, Rio de Janeiro, 2010. Disponível em: <https://www.gapcongressos.com.br/ eventos/z0070/trabalhos/final_249.pdf> Acesso em: 12/08/ 2020.

O Estilo de Vida da Terceira Idade Contemporânea no Brasil. Serviço de Proteção ao Crédito (SPC Brasil); Confederação Nacional de Dirigentes Lojistas (CNDL), 2016. Disponível em: < http://www.cndl. org.br/wp-content/uploads /2016/10/Apresenta\%C3\%A7\%C3\%A3o-Consumo-de-Idosos-_-Estilo-de-Vida-e-Tecnologia-v8.pdf.> Acesso em: 20/08/2020.

OliveIRA, D.A. Terceira Idade e Tecnologia: A Identidade dos Idosos a Partir do Consumo na Web. Universidade Cásper Líbero. São Paulo. 2014. Disponível em: <http://congreso.pucp.edu.pe/ alaic2014/wp-content/uploads/2013/09/GT10-Diego-de-Oliveira.pdf>. Acesso em 08/09/2020.

O "X" da Questão - Pesquisa Grupo Locomotiva (2017). Disponivel em: $<$ https://www.ilocomotiva. com.br/single-post/2017/07/27/O-X-da-quest\%C3\%A3o.> Acesso em 29/08/2020.

Pesquisa Terceira Idade 2018. SPC Brasil. Disponível em: < https://www.spcbrasil.org.br/pesquisas/ pesquisa/5290> Acesso em 20/08/2020.

Pesquisa Sobre o Uso das Tecnologias de Informação e Comunicação nos Domicílios Brasileiros - TIC DOMICÍlLIOS. Núcleo de Informação e Coordenação do Ponto BR (NicBR). São Paulo. 2018. Disponível em: <https://cetic.br/media/docs/publicacoes/2/tic_dom_2017_livro_eletronico.pdf> Acesso em 16/08/2020.

\section{Principais Interesses nas Redes Sociais}

Disponível em: <https://cdn2.hubspot.net/hubfs/355484/Ebooks\%20MKTC/Social\%20Media\%20 Trends\%202018.pdf?t $=1542483912554 \& u t m \_s o u r c e=h s \_a u t o m a t i o n \& u t m \_m e d i u m=e m a i l \& u t m \_c o n-$

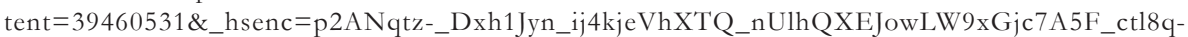
95j0xl3YWk-uwDVF101YqLcGPoDLKcycymEKAEeJ8xxw\&_hsmi=39460531.> Acesso em: 14/09/2020.

Relógio da população. Disponível em: https://countrymeters.info/pt. Acesso em: 14/09/2020.

RockContent. Pesquisa Social Media Trends 2018. Disponível em: <https://cdn2.hubspot.net/hubfs/355484/Ebooks\%20MKTC/Social\%20Media\%20Trends\%202018.pdf?t=1542483912554\&utm_source=hs_automation\&utm_medium $=$ email\&utm_content $=39460531 \& \_$hsenc $=$p2ANqtz-_Dxh1Jyn_ij4kjeVhXTQ_nUlhQXEJowLW9xGjc7A5F_ct18q95j0x13YWk-uwDVF101YqLcGPoDLKcycymEKAEeJ8xxw\&_hsmi=39460531> Acesso em 17/08/2018.

SENADO FEDERAL. Constituição da República Federativa do Brasil: Artigo 196. Brasília, DF, 1988.

SENADO FEDERAL. Portaria no 3.916, de 30 de outubro de 1998. Diário Oficial [da] União, Brasília, DF, 1998. Disponível em: <http://bvsms.saude.gov.br/bvs/saudelegis/gm/1998/prt3916_30_10_1998. html>. Acesso em: 28/08/2020.

SINTOX - Sistema Nacional de Informações Toxico Farmacológicos < https://sinitox.icict.fiocruz.br/ sites/sinitox.icict.fiocruz.br/files//Brasil11_1.pdf> Acesso em: 12/09/2020. 
Sociedade Brasileira de Anestesiologia. Manual de Conduta nas Redes Sociais. Disponível em: $<$ https:// www.google.com $/$ search?q=anestesiologia $\% 2$ Bmanual-de-conduta-nas-midias-sociais-2+ed.pdf\&sa $=$ X\&biw $=1517 \&$ bih $=670 \& \mathrm{tbm}=$ isch\&source $=$ iu\&ictx $=1 \&$ fir $=$ lzXQ5XqxixSzdM $\% 253 \mathrm{~A} \% 252 \mathrm{CeFIrgTnpBu}-$ Vt3M\%252C_\&vet=1\&usg=AI4_-kQwws7M3APYT0WpxIEORgIoQTBmoQ\&ved=2ahUKEwir2Lmwo6HkAhU_IrkGHUQADHgQ9QEwCHoECAkQDA\#imgrc=X4VCSoLL7FlyXM:\&vet=1> Acesso em: 26/08/2020.

\section{Razões de Utilização das Redes Sociais de Saúde}

The Sociology of Caregiving. p.202. Disponível em: < https://scholar.google.com.br/scholar?hl=p$\mathrm{t}$-BR\&as_sdt $=0 \% 2 \mathrm{C} 5 \&$ as_vis $=1 \& \mathrm{q}=\mathrm{The}+$ Sociology + of + Caregiving. + p. $202+\& \mathrm{btnG}=>$ Acesso em: $14 / 09 / 2020$.

SOUZA, F.S.; GOMES, A.O. Conflitos de Gerações no Ambiente de Trabalho: O Caso da Procuradoria Geral do Trabalho (PGT). Revista Foco.

V.11, n², p.181-206, mar./jun. 2018. Disponível em: <http://revistafocoadm.org/index.php/foco/article/ view/531> Acesso em: 12/09/2020.

\section{Usuários de Redes Sociais na América Latina}

Disponível em: <https://canaltech.com.br/redes-sociais/brasil-e-o-pais-que-mais-usa-redes-sociais-na-america-latina-70313/.> Acessado em: 14/09/2020. 